\title{
THALASSEMIA-INDUCED OSTEOPOROSIS AND BIPHOSPHONATE TREATMENT: A SYSTEMATIC REVIEW AND META-ANALYSIS
}

Athanasios Tsartsalis ${ }^{1}$, George I. Lambrou ${ }^{2}$, Christos Savvidis ${ }^{3}$, George P. Chrousos ${ }^{2}$ and Antonis Kattamis ${ }^{2}$

1.Department of Endocrinology, Diabetes and Metabolism, Naval Hospital of Athens, Greece

2.First Department of Pediatrics, University of Athens

3. Department of Endocrinology, Hippokration Hospital, Athens Greece

\section{OBJECTIVES}

Osteopenia and osteoporosis develop with aging in Thalassemia patients despite the progress in clinical treatment and therapy. Bisphosphonates, which are potent inhibitors of osteoclastic bone resorption, have been recently used to correct the bone abnormality in Thalassemia with encouraging results. Bisphosphonates are a class of drugs that prevent the loss of bone mass, used to treat osteoporosis and similar diseases. They are the most commonly prescribed drugs used to treat osteoporosis. Bisphosphonates act by inhibiting osteoclastic recruitment and maturation, preventing the development of monocyte precursors into osteoclasts, inducing osteoclast apoptosis and interrupting their attachment to the bone. The scope of the present work was to meta-analyze available data concerning the role of bisphosphonates in thalassemia-induced osteoporosis.

\section{METHODS}

A search was carried out to find relevant studies and reviews published up to date. The database used was PubMed. The Mesh terms used were: bisphosphonate, thalassemia and/or anemia, osteoporosis, which yielded 33 results. From those 22 has been selected for further processing as more relevant to the topic of study. Two independent reviewers read, reviewed and selected the articles. A third reviewer decided whether or not an article would be included if the first two reviewers did not agree The information was extracted from all studies by the same person and the results, i.e. cumulative risks, were obtained directly from the studies when available, extrapolated from the information available in the study (mainly text or curves). The SE could be only derived when the p-value, StdDev or the CI was reported in the study. Therefore, only studies with this information available could be pooled. Statistical heterogeneity of data included in the pooled analysis was estimated by calculation of the Cochran's Q statistic value as well as heterogeneity and inconsistency quantification was estimated using the $\mathrm{I}^{2}$ test.

Data have been pre-processed with the MS Excel environment and data analysis was performed using the MATLAB ${ }^{\circledR}$ (The Mathworks Inc., Natick MA) simulation environment.

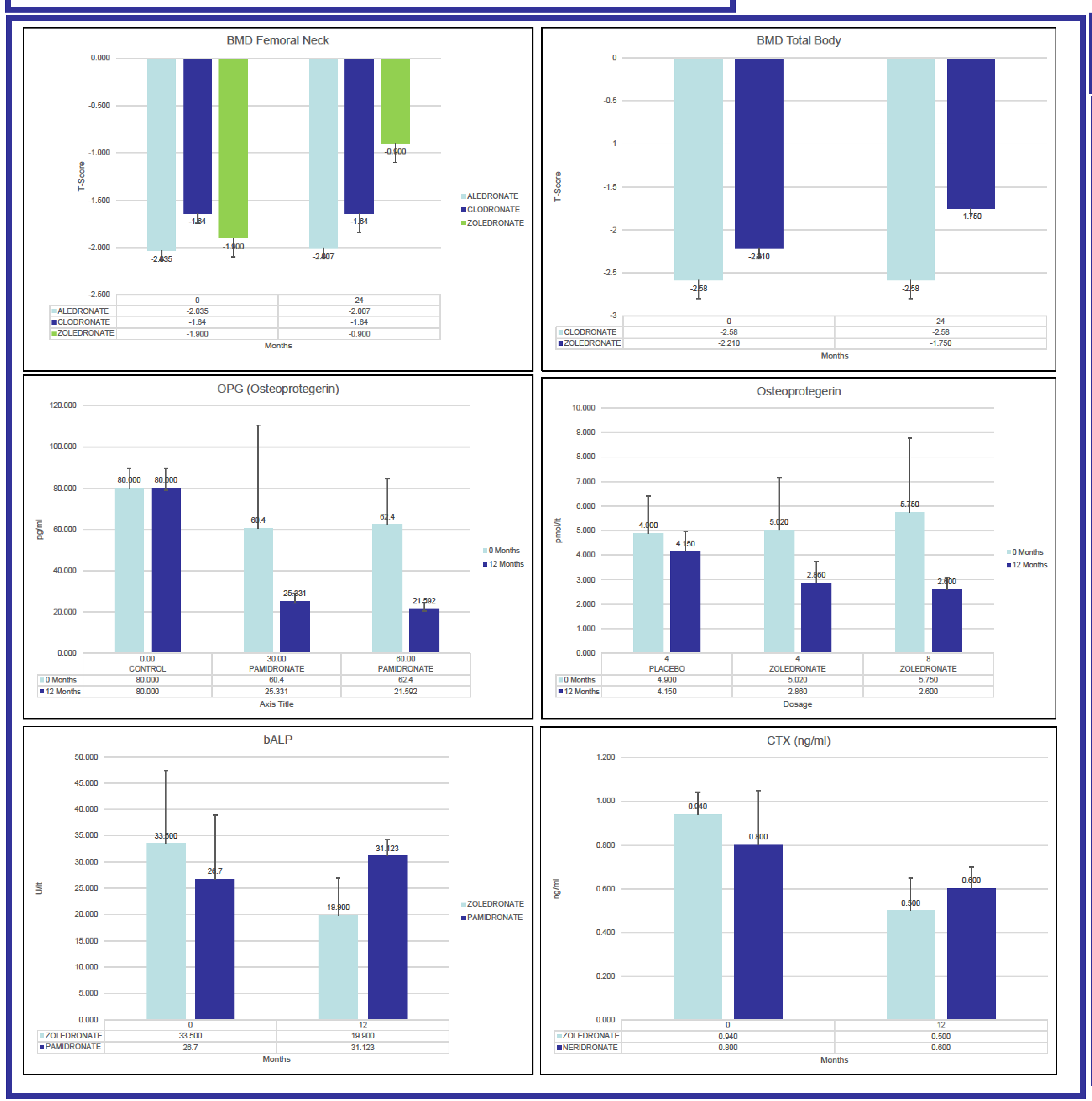

RESULTS

Based on the meta-analysis of selected studies it appeared that bisphosphonates could prove an effective treatment for osteoporosis. In particular, it appeared that zoledronic acid was effective in the treatment of osteoporosis, while clodronate did not appear to be equally effective. In total, it appeared that bone mineral density as well as markers of bone remodeling (CTX, bALP, difference in OPG etc.) were higher in the bisphosphonate-treatment groups as compared to the placebo group. In particular, Bone Mineral Density (BMD) Femoral Neck (FN) manifested improvement with the zoledronic acid as compared to alendronate and clodronate. Further on, BMD total body increased with Zoledronate as compared to clodronate. Similar results appeared with pamidronate and zoledronate with respect to osteoprotegerin. Interestingly, bone alkaline phosphatase (bALP) as well as C-telopeptide of collagen type I (CTX) manifested better results with zoledronate as compared to pamidronate and neridronate respectively. Both markers (ALP and CTX) are significant molecules of bone turn-over and the effects of biphosphonates appear to be important in the development of those factors.

\section{CONCLUSIONS}

Cross-examination of several studies suggests that bisphosphonates are to be considered as a therapeutic option for the treatment of thalassemia-induced osteoporosis. From all comparisons made across all studies it appeared that bisphosphonates can be considered as an effective therapy for thalassemia-induced osteoporosis and at the same time it appears that they also can play a positive role in the treatment of osteoporosis. Additionally, all studies agree that the use of bisphosphonates could be beneficial for the treatment of osteoporosis in thalassemia patients. Also, all studies show that the most probable potent factor could be the zoledronic acid, yet the other treatments appeared to also have an effect in osteoporosis.

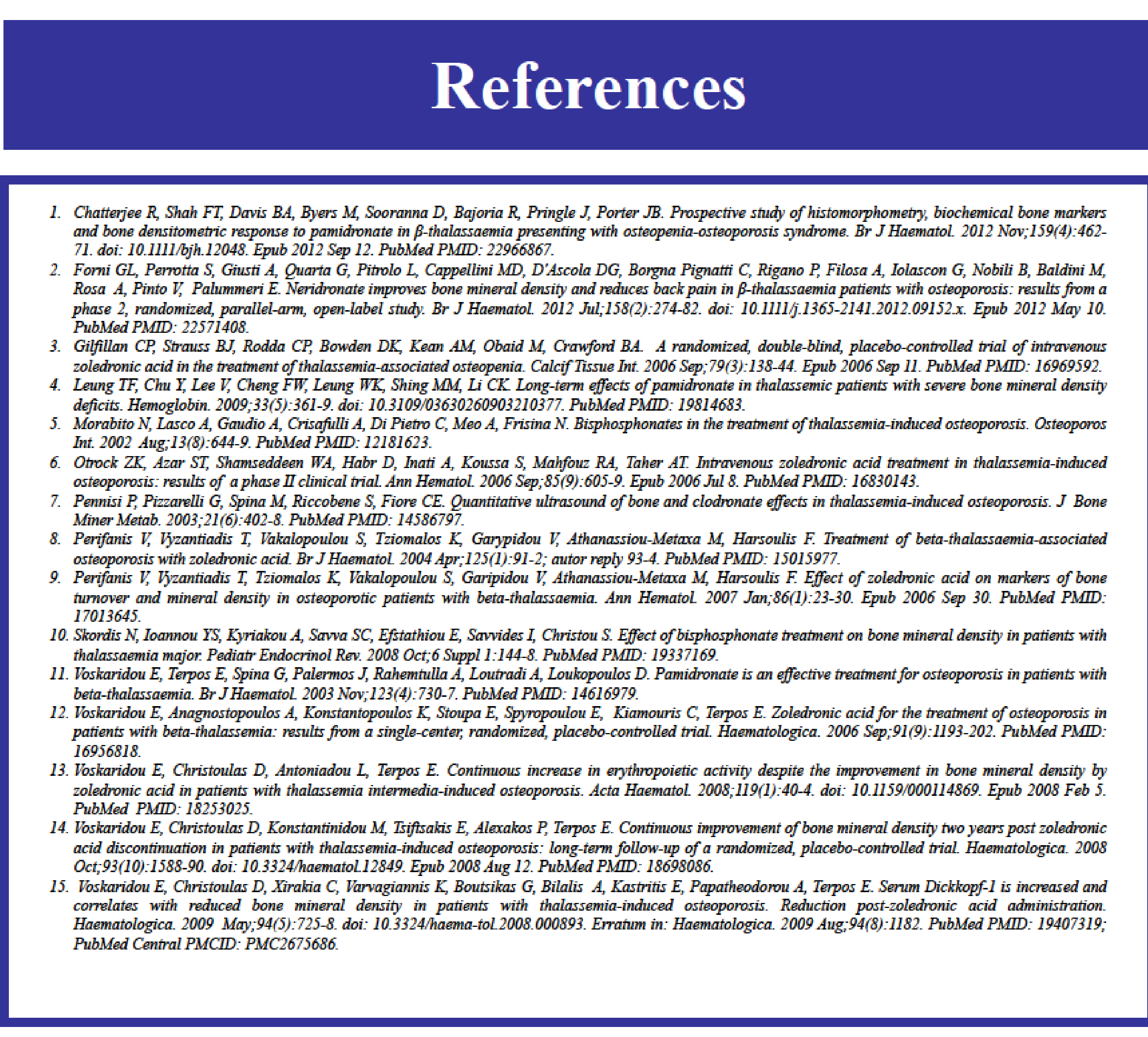

